\title{
Long Memory and Fractality in the Universe of Volatility Indices
}

\author{
Bikramaditya Ghosh $\mathbb{D}^{1}$ and Elie Bouri ${ }^{2}$ \\ ${ }^{1}$ SIBM, Symbiosis International University, Bangalore, India \\ ${ }^{2}$ School of Business, Lebanese American University, Beirut, Lebanon \\ Correspondence should be addressed to Bikramaditya Ghosh; b.ghosh@sibm.edu.in
}

Received 1 November 2021; Accepted 6 January 2022; Published 20 January 2022

Academic Editor: Andreas Pape

Copyright (c) 2022 Bikramaditya Ghosh and Elie Bouri. This is an open access article distributed under the Creative Commons Attribution License, which permits unrestricted use, distribution, and reproduction in any medium, provided the original work is properly cited.

\begin{abstract}
Unlike previous studies that consider the Chicago Board of Options Exchange (CBOE) implied volatility index (VIX), we examine long memory and fractality in the universe of nine CBOE volatility indices. Using daily data from October 5, 2007, to October 5, 2020, covering calm and crisis periods, we find evidence of long memory and fractality in all indices and a change in the degree of volatility persistence, which points to inefficiency. The long memory of the SKEW index is strong before the onset of three crisis periods, but eases afterwards. The findings provide new insights that matter to investment decisions and trading strategies.
\end{abstract}

\section{Introduction}

Within the basis of the efficient market hypothesis (EMH) of Malkiel and Fama [1], the weak form efficiency hypothesis points to the inability of investors to exploit information from historical prices to earn consistent abnormal profits. However, the presence of long-term memory is often shown in financial asset returns [2-4], indicating that prices do not respond immediately to the flow of information and that shocks to the volatility process tend to persist. Accordingly, past returns can be used to predict current returns, which challenges the weak form efficiency hypothesis. Furthermore, evidence suggests that biases driven by investor heuristics often cause stock markets to drift from being efficient $[5,6]$. Such biases become prominent when investors and fund managers behave irrationally, regardless of their knowledge and expertise, which suggests the importance of mass psychology in driving market inconsistency $[5,6]$. Interestingly, implied volatility indices are established to reflect investor psychology, and, thus, the volatility tracking and the pattern identification become quite pertinent. In this regard, the fractal market hypothesis (FMH), developed by Peters [7], relies upon investor behaviour contrary to the EMH that considers investors to be rational (previous studies consider the presence of long-term memory properties to make inferences in favour of the FMH and thus the possibility of predicting stock prices). In this regard [8], the Hurst exponent is widely used to measure long-term memory properties through its ability to quantify the degree of persistence of similar price change patterns. The FMH was considered during the dot-com bubble of 2001 and the global financial crisis in 2008 (Karp and Van Vuuren, 2019). Studies examining the presence of long memory and efficiency in the wide universe of volatility indices are very limited. For example, Caporale et al. [9] have showed the persistence in the Chicago Board Options Exchange (CBOE) VIX. Bogdan et al. [10] have studied the efficiency of the VIX index and provided evidence of substantial change in its efficiency during 2020. However, understanding the dynamics of volatility is important for decisions regarding equity valuation, hedging, and risk management, especially given the wide universe of volatility indices that has been extended to include not only the wellestablished Chicago Board Options Exchange CBOE VIX but also other important indices for leading US equity indices (the VXN for the NASDAQ 100, the VXO for the S\&P 100 , the VXD for the Dow Jones Industrial average, the RVX for the Russell 2000, and the OVX for the crude oil price) as well as the volatility of the VIX (VVIX), the Premium Strategy Index (VPD), and the SKEW index. While volatility 
indices are time-varying, a key question concerns the persistence of their levels and the evolution of their efficiency. Our goal is to extend the scarce literature by Hurst exponent using the autoregressive fractionally integrated moving average (ARFIMA) model considering the wide universe of volatility indices covering VIX, VXN, VXO, VXD, RVX, VPD, OVX, VVIX, and SKEW. We do that for various defined samples of daily data covering the period October 5 , 2007, to October 5, 2020, which includes tranquil and crisis periods. Methodologically, we employ the Hurst exponent based on the autoregressive fractionally integrated moving average (ARFIMA) model and the fractal dimension as an additional confirmatory tool.

Our current study is related to studies considering market persistence and efficiency in stock market indices $[2-4,11]$, cryptocurrencies [12], and economic uncertainties [13]. For implied volatility indices, Caporale et al. [9] have shown first empirical evidence on the persistence in the CBOE VIX. Our paper is more comprehensive given its inclusion of more CBOE volatility indices such as the VXN, VXO, VXD, RVX, VPD, OVX, VVIX, and SKEW, as well as its coverage of the COVID-19 outbreak period. It extends our limited knowledge of the behaviour of implied volatility indices by considering the persistence of a large set of volatility indices using long-memory methods. This is informative for policymakers and practitioners regarding market efficiency and predictability. In fact, evidence of predictability reflects evidence of market inefficiency [9], which can be exploited by crafting trading strategies to earn abnormal profits in $\mathrm{CBOE}$ volatility indices that are mostly tradeable. Furthermore, evidence of long-memory properties matters to derivative traders and risk managers [14].

\section{Data and Methodology}

2.1. The Dataset of Volatility Indices. The CBOE followed the market clues back in 1973 and introduced its first implied volatility index (VIX) for S\&P 500 in 1993. The VIX was perfected in the next decade, with a change in its methodology. Known as the market fear gauge, the VIX has predictive power over stock returns (e.g., $[15,16])$. Subsequently, the CBOE launched NASDAQ-100 based VXN at the onset of the dot-com bubble back in 2001, and later on it developed the RVX as a near term implied volatility index for the Russell 2000 in 2004 and the VXD for the Dow Jones Industrial Average in 2005. The VXO followed soon, reflecting the implied volatility of the S\&P 100. Interestingly, the CBOE developed the SKEW or Black Swan Index from the tail risk of the S\&P 500. VVIX measures the volatility in VIX itself. OVX was developed in 2007 to predict volatility related to crude oil. VPD, or the Premium Strategy Index with a modified VIX (auto-account sell on every 1 month), was also introduced in 2007. Thus, this basket of volatility measures covers virtually all the known volatility universe. Hence, long-memory investigation of such a universe could lead to persistence.

Daily closing levels of nine volatility indices (VIX, VXN, VXO, VXD, RVX, VPD, OVX, VVIX, and SKEW) are extracted from the CBOE (http://www.cboe.com). The sample period is October 5, 2007, to October 5, 2020, determined by data availability, yielding 3,354 data points for each index. The rationale behind the selection of these nine volatility indices is the difference not only in their underlying assets (e.g., Russell 2000, DJIA, S\&P 500, S\&P 100, NASDAQ, Crude Oil ETF, Premium Strategy, Tail Risk Index, and volatility of volatility index) but also in their construction, for example, the Premium Strategy index and the Tail Risk Index.

Figure 1 depicts the time plot of the nine volatility indices under study. Notably, there is a large spike in the levels of VIX, VXN, VXO, VXD, RVX, OVX, and VVIX around the COVID-19 outbreak in early 2020, whereas a drop is shown for the Capped VIX Premium Strategy Index (VPD) around that time, which reflects the performance of a strategy that overlays a sequence of short, one-month VIX futures. The SKEW index, which generally fluctuates between 100 and 150 points, reached the 150 level a few times around the COVID-19 outbreak, reflecting the overall fear in the US market and thus the fact that the implied volatilities for the out-of-the-money (OTM) puts were much higher than those of the OTM calls.

In our empirical analysis, we consider the pre-COVID19 outbreak period covering March 1, 2019, to December 31, 2019 (212 daily observations) and the during COVID-19 outbreak period covering January 1, 2020, to November 2, 2020 (212 daily observations). Accordingly, two windows of equal length of 212 daily observations in the pre-COVID-19 and during COVID-19 periods are put to test, which help unravel the degree of long memory during the pandemic.

The summary statistics of the level series for the nine volatility indices are presented in Table 1. Apparently, the most (least) volatile index is the VPD (VIX). All indices are non-normally distributed as evidenced by the Jarque-Bera statistics, which points to the suitability of applying the Hurst exponent. However, SKEW tends to be mesokurtic in nature. Skewness and kurtosis values are largest for the OVX, which might be due to its unprecedented spike when crude oil prices collapsed to negative territories during the COVID-19 outbreak and the oil price war between Saudi Arabia and Russia. Except for VPD, all volatility series are stationary as shown by the augmented Dickey-Fuller (ADF) test. Therefore, we conduct the analysis of the VPD based on its first difference to ensure stationarity (unreported results from the ADF test show that the first differences of the VPD index are stationary at the $1 \%$ level of significance), whereas for the rest of indices the analysis is conducted with level series.

2.2. Methodology. The ARFIMA model is a parametric method used to examine the long-memory trait in time series $[14,17]$. In the ARFIMA $(p, d, q)$ model, $p$ represents the lag of autoregression, $q$ represents the lag of moving average, and $d$ is the fractional integrating parameter. The ARFIMA $(p, d, q)$ model can be expressed as a generalization of the ARIMA model as follows: $\varepsilon_{t}=m_{t} \sigma_{t}$, where $m_{t} \sim N(0,1)$ 
VIX

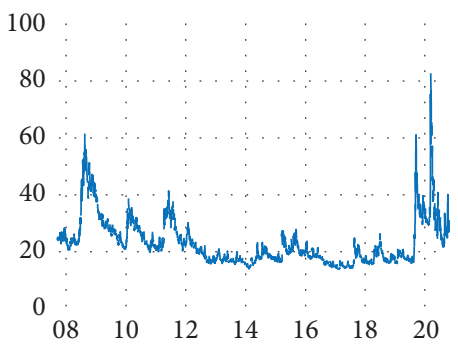

VXD
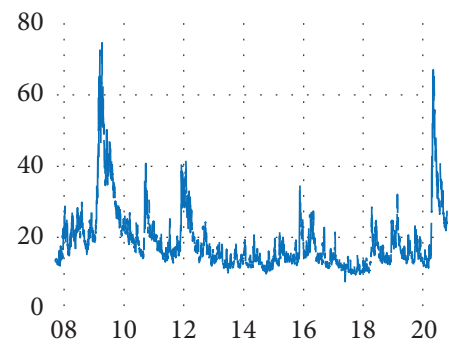

OVX

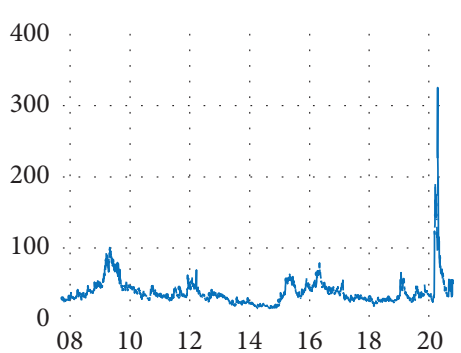

VXN

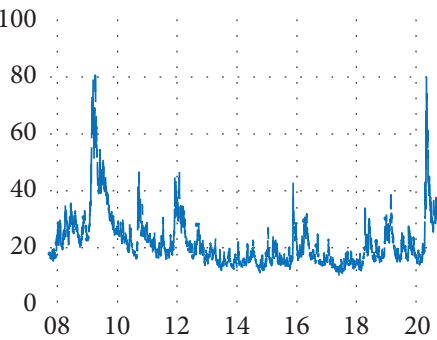

RVX

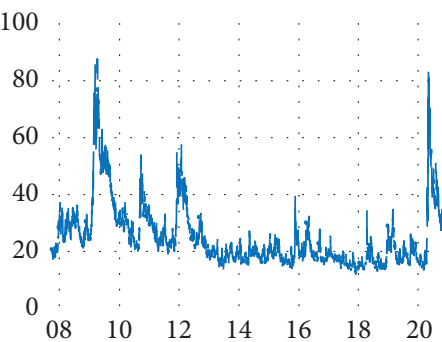

VVIX

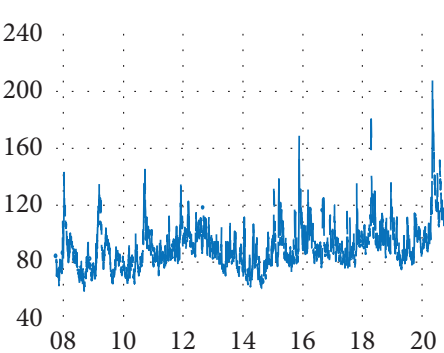

VXO

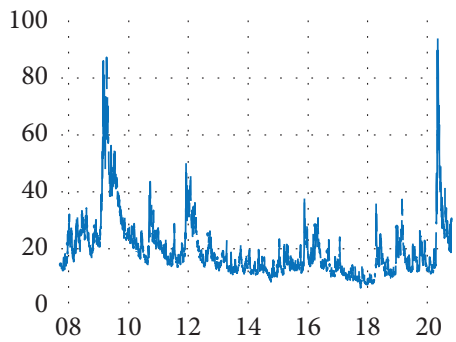

VPD

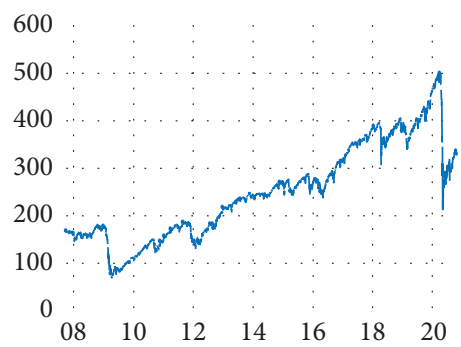

SKEW

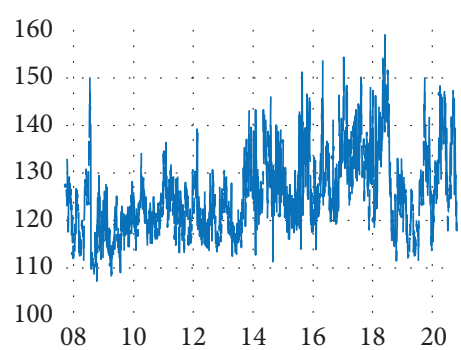

Figure 1: Levels of the nine volatility indices from October 5, 2007, to October 5, 2020.

TABLE 1: Descriptive statistics for the levels of the nine CBOE volatility indices.

\begin{tabular}{lcccccccc}
\hline Volatility index & Mean & Max & Min & SD & Skewness & Kurtosis & Jarque-Bera & ADF test \\
\hline VIX & 23.148 & 82.690 & 13.750 & 8.199 & 1.934 & 8.360 & $6106.486^{*}$ & $-4.468^{*}$ \\
VXN & 22.046 & 80.640 & 10.310 & 9.324 & 2.318 & 10.449 & $10758.400^{*}$ & $-5.022^{*}$ \\
VXO & 19.790 & 93.850 & 6.320 & 10.650 & 2.498 & 11.674 & $14004.380^{*}$ & $-5.067^{*}$ \\
VXD & 18.877 & 74.600 & 7.580 & 8.867 & 2.442 & 10.562 & $11323.660^{*}$ & $-4.212^{*}$ \\
RVX & 24.975 & 87.620 & 11.830 & 10.965 & 2.037 & 8.020 & $5842.102^{*}$ & $-4.355^{*}$ \\
VPD & 248.506 & 500.010 & 69.260 & 99.828 & 0.367 & 2.246 & $154.767^{*}$ & -1.232 \\
OVX & 37.975 & 325.150 & 14.500 & 19.209 & 4.463 & 39.260 & $194874.000^{*}$ & $-5.333^{*}$ \\
VVIX & 90.860 & 207.590 & 59.740 & 15.508 & 1.527 & 8.245 & $5147.311^{*}$ & $-9.48^{*}$ \\
SKEW & 125.401 & 159.030 & 107.230 & 8.285 & 0.702 & 3.146 & 278.5628 & $-8.681^{*}$ \\
\hline
\end{tabular}

Note: the sample period is October 5, 2007, to October 5, 2020, yielding 3,354 daily observations. ${ }^{*}$ denotes significance at the $5 \%$ level. ADF test is conducted with intercept.

$$
\phi(B)(1-B)^{d} Y_{t}=\theta(B) \varepsilon_{t},
$$

where $Y_{t}$ is the state output represented by the time series, $\Phi$ $(B)$ and $\theta(B)$, respectively, represent autoregressive and moving average operators, with $\mathrm{B}$ being the lag operator; $d$ is the fractional parameter that varies between -0.5 and +0.5 , which is also called the memory parameter because it regulates the long-memory property of the time series; and $\varepsilon_{t}$ represents the white noise term. According to Hosking [17], whenever $-0.5<d<0.5$, then $Y_{t}$ is mean reverting. We use a fixed window method (calendar year) to calculate the longmemory parameter with the local Whittle estimator of Robinson [18]. The strength of the long-range dependence is calculated by the fractional integration parameter $d$.

Furthermore, we calculate the Hurst exponent $H \approx d+$ 0.5 [19] (as argued by Torre et al. (2007), "ARFIMA modelling provides an interesting method for estimating fractal exponents") to evaluate the long-memory intensity. $H$ varies 


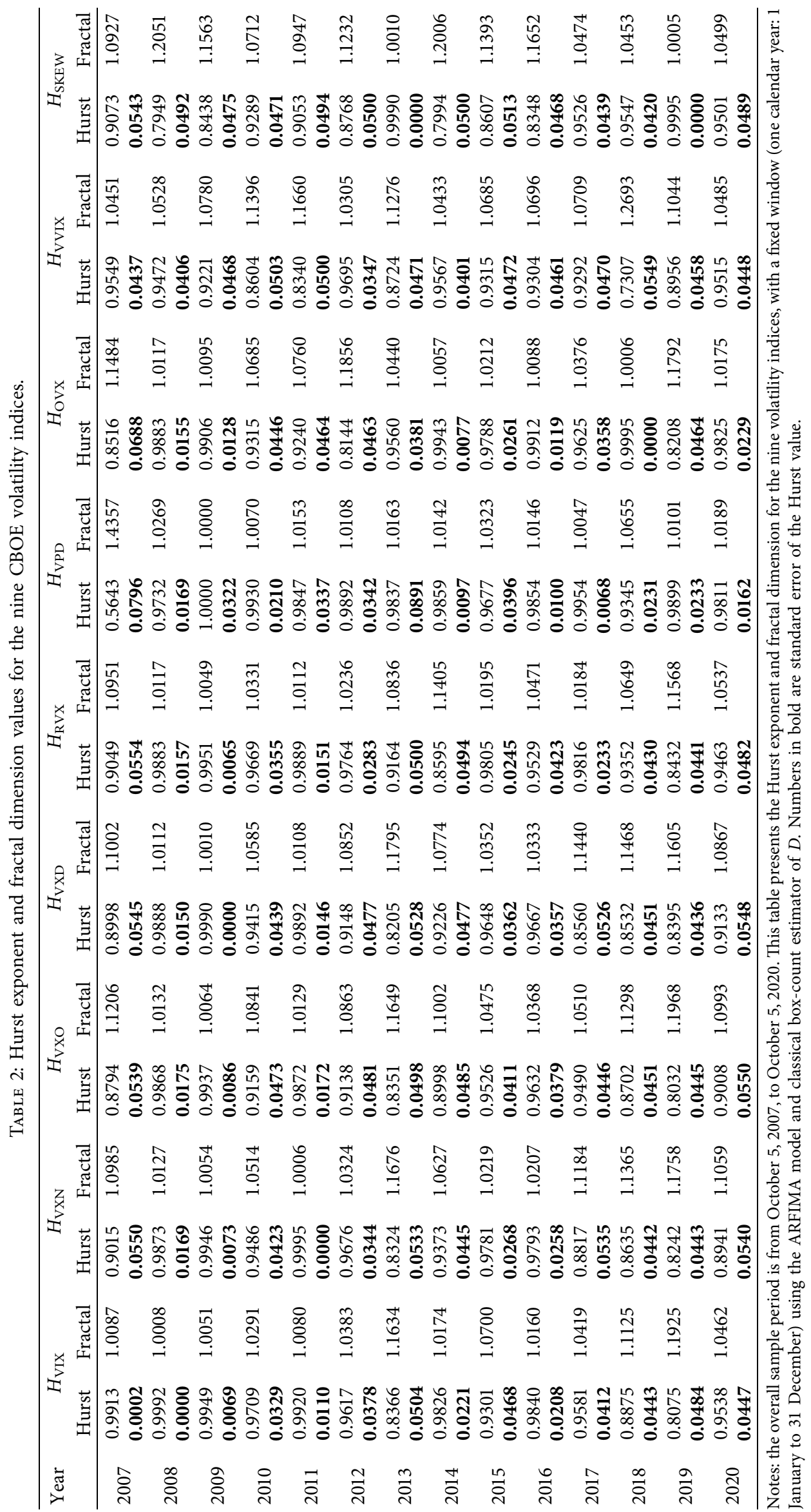




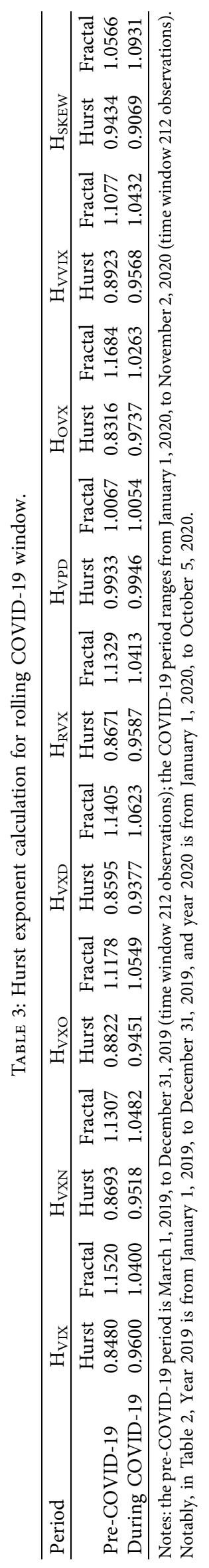


between 0 and 1 and thus the Hurt exponent can have four conditions as follows:

If $0.5<H \leq 1$, then the time series is persistent and shows evidence of long memory, which contradicts the EMH. The higher the $\mathrm{H}$ value, the higher the persistence and long memory.

If $0<H<0.5$, then the time series is anti-persistent (i.e., has a short memory). Such a condition indicates fast changes in the direction of movements of the series.

If $H=0$, then the time series exhibits no long-range dependence.

If $H=0.5$, then the time series follows a random walk, which supports the EMH. Notably, the Gaussian distribution is observed with the series having no memory. However, it is difficult for the process to achieve $H=0.5$, which is merely a point rather than a range.

We have also calculated fractal dimension $(D)$ as an additional confirmatory tool, widely accepted in long-memory research. Fractal dimension or Hausdorff dimension $(D)$ is widely considered as an indicator for surface roughness. It functions as an estimator of the "short-range memory" or "local" memory. It has been well documented that, for any selfaffine processes, the fractal dimension is linked to the longrange dependence or long memory of the underlying series. The relationship of Hurst exponent and fractal dimension is linear and represented by $D=2-H$. Furthermore, $D=1.5$ is denoted as random walk or an indicator of market efficiency. Market efficiency gets violated if the condition is not satisfied. Moreover, $D=1.5$ signifies no local trend as well. The classical box-count estimator of $D$ has been put into use here. It has conditions such as the following: if $N(\varepsilon)$ denotes the number of boxes required at scale $\varepsilon$, the box-count estimator equals the slope in an OLS fit of $\log (N(\varepsilon))$ on $\log (\varepsilon)$ [20].

\section{Empirical Results}

We present in Table 2 the values of the Hurst exponent $(H)$ and the standard errors (SE) of the nine volatility indices. They range from 0.56431 to 0.99999 , showing that the extent of longmemory changes within a short range. Past levels in the volatility index have a significant impact on current levels, suggesting that correlations between levels decay very slowly. The results indicate the presence of the long-memory effect in all volatility indices (given that the estimates of the Hurst exponent are larger than 0.5 ). Persistence in the volatility indices is present over the years; however, the degree of persistence changes over time. Accordingly, the results indicate that none of the volatility indices follows a random walk (in fact, none of the nine volatility indices under study has an $H$ value below 0.55 for the entire observation period from October 5, 2007, to October 5, 2020).

For the VPD, the Premium Strategy Index with auto adjustment remains the most persistent with an $H$ value very close to 1 throughout the sample period 2008-2020. Another interesting observation is that almost all the volatility indices are above the extremely high persistence zone $(H>0.9)$ during the 2008 global financial crisis. The only exception is SKEW, called the Black Swan Index, which is based on the slope of implied volatility. A similar pattern is observed during the BREXIT announcements and multiple bailouts of Greece during 2014-2016. This pattern however breaks in 2020 (amid the COVID-19 outbreak). Hence, the persistence and extent of long memory in SKEW is relatively less than the other volatility indices during financial crisis periods. The Hurst value for the SKEW clocks marginally below 0.8 on two occasions. Interestingly, both are during financial crisis periods (2008 and 2014). Another important observation is that the Hurst value of SKEW is in the extreme zone, clocking well above 0.9, just before a financial crisis on both occasions (2007 and 2013). Even before the COVID-19 pandemic, it clocked close to 1 . This might imply that the long memory of SKEW holds the clue to future crisis periods. Usually, extremely high Hurst values coincide with the peaks of crises [9]. The SKEW index, which tracks the S\&P 500 tail risk, computes the probability of a S\&P 500 move of $2 \sigma$ from its mean value 30 days in advance; for example, when the SKEW is 130 , a $2 \sigma$ deviation has a probability of $10.4 \%$. The sudden drop of the Hurst exponent of the SKEW in various periods (the global crisis of 2008, BREXIT 2016, and the onset of the pandemic in 2020) indicates a sudden change in probability of $2 \sigma$ deviation. The probability is either increasing or decreasing drastically. Results from other volatility indices indicate that their levels are positively correlated, generating persistence and long memory throughout the sample. As for the SE of the Hurst exponent, they are extremely low on all occasions, indicating the robustness of Hurst exponent measurements.

Table 2 also indicates a $D \neq 1.5$ for all one twenty-six outcomes over nine volatility indices (from October 5, 2007, to October 5,2020), signifying that the idea of EMH is getting defenestrated entirely. In fact, the observations are quite similar even in COVID-19 subsamples (see Table 3) under consideration. Since volatility is fundamentally derived from various financial asset classes, hence, it can be said that financial market efficiency suffered during COVID-19 as well.

In Table 3, we compute the Hurst exponent for the preCOVID-19 and during COVID-19 periods using a rolling window approach that tends to provide more reliable results. The results show that, during the COVID-19 outbreak period, $\mathrm{H}_{\text {SKEW }}$ drifted marginally away from 1 , whereas other Hurst values progressed towards 1 . Otherwise, the major patterns remained consistent with the findings from Table 2. Notably, more than $70 \%$ of our selected sample period covers periods of turmoil that are diverse in nature. For example, it includes the build-up and crash period around the 2008 global financial crisis, the EU sovereign Debt crisis, and the COVID-19 outbreak. This can be shown in the Hurst values that exhibit high persistence during these crisis periods, intuitively indicating bubbles and anti-bubbles. Our results draw parallel with previous studies of repute though with only the VIX $[9,10]$.

\section{Conclusion}

While evidence for long-term memory in the US stock market is well recognized (e.g., [2]), much less is known about the various implied volatility indices. To address this gap, we examine the presence of long-memory properties in 
nine CBOE volatility indices (VIX, VXN, VXO, VXD, RVX, VPD, OVX, VVIX, and SKEW) to make inferences regarding the FMH and the possibility of applying prediction models based upon past volatility. The main results are summarized as follows. Firstly, the consistent presence of long memory in various volatility indices supports the FMH. Past volatility certainly provides information about future prediction. Secondly, these empirical findings provide a theoretical premise for trading strategies. Evidence of the consistent presence of long memory points to the utility of applying trend-based trading strategies such as moving average convergence divergence (MACD). Thirdly, the SKEW might be used as a predictor of various probable crises (both financial and non-financial). Fourthly, the relative instability in long-memory traits is visible in all volatility indices. Hence, trading strategies might need to switch periodically for more consistent performance.

Our results supporting the presence of a degree of persistence are extremely useful to both investors and policy-makers for identifying herding, and to traders opting for generating abnormal returns using trend-based techniques such as the MACD. We found true long memory in all the cases (i.e., persistence coupled with mean reverting feature). This means permanent policy shocks are required rather than random policy shocks, as suggested by some eminent studies $[21,22]$. Circuit filters, deployed in the stock markets, can be truncated for a reasonably long period of time, in order to contain volatility within specified limits. Option trading could be restricted for a long period as well.

Notably, the results show that long memory is persistent through all crisis phases such as the global financial crisis (2007-2008), BREXIT (2016), and lately COVID-19 (2020) in an extremely consistent manner. Hurst exponent did not decrease drastically across all nine volatility indices even during relatively calmer periods such as 2012 to 2014 . This observation indicates towards embedded speculative bubble formation (of various degrees) on all occasions under consideration. Intuitively, if the near-term (e.g., last 15 days) Hurst exponent is larger than the comparatively midterm (e.g., last 30 days), then this indicates an increase in the degree of herd, which might be understood in the context of panic selling during crisis periods. If short selling is possible under regulations, it could be well the trader's call. Suppose the 15-day Hurst is lower than the 30-day Hurst; this indicates in a steady market with surging investor sentiment index that a trend reversal is around the corner and that bull market players have eased or ceased their constant buying. Given the emergence of the SKEW as a potent indicator of crisis prediction, it deserves further in-depth analysis via the application of suitable prediction models. SKEW essentially reflects the tail risk of S\&P500 through implied volatility of OTM strikes. The higher the SKEW (closer to 150), the higher the chances of a probable Black Swan event. In fact, SKEW-Hurst enjoyed a strong negative correlation with VIXHurst (-0.74). A rising SKEW (persistent) coupled with a falling VIX (persistent) is considered bearish in equity markets, encouraging short selling (provided permitted by the regulators). Our results showed such trends, especially in 2009, 2017, and 2020. That issue of SKEW-VIX relationship is left to future studies [20].

\section{Data Availability}

Access to data is restricted.

\section{Conflicts of Interest}

The authors declare that they have no conflicts of interest.

\section{References}

[1] B. G. Malkiel and E. F. Fama, "Efficient capital markets: a review of theory and empirical work," The Journal of Finance, vol. 25, no. 2, pp. 383-417, 1970.

[2] J. Alvarez-Ramirez, J. Alvarez, E. Rodriguez, and G. Fernandez-Anaya, "Time-varying Hurst exponent for US stock markets," Physica A: Statistical Mechanics and Its Applications, vol. 387, no. 24, pp. 6159-6169, 2008.

[3] L. B. Martinez, M. B. Guercio, A. F. Bariviera, and A. Terceño, "The impact of the financial crisis on the long-range memory of European corporate bond and stock markets," Empirica, vol. 45 , no. 1, pp. 1-15, 2018.

[4] S. Sadique and P. Silvapulle, "Long-term memory in stock market returns: international evidence," International Journal of Finance \& Economics, vol. 6, no. 1, pp. 59-67, 2001.

[5] H. Shefrin, Beyond Greed and Fear: Understanding Behavioral Finance and the Psychology of Investing, Oxford UK, 2000.

[6] R. J. Shiller, "From efficient markets theory to b finance," The Journal of Economic Perspectives, vol. 17, no. 1, pp. 83-104, 2003.

[7] E. E. Peters, Fractal Market Analysis: Applying Chaos Theory to Investment and Economics, John Wiley \& Sons, New York, 1994.

[8] A. Karp and G. Van Vuuren, "Investment implications of the fractal market hypothesis," Annals of Financial Economics, vol. 14, no. 01, Article ID 1950001, 2019.

[9] G. M. Caporale, L. Gil-Alana, and A. Plastun, "Is market fear persistent? A long-memory analysis," Finance Research Letters, vol. 27, pp. 140-147, 2018.

[10] D. I. M. A. Bogdan, Ş. M. Dima, and I. O. A. N. Roxana, "Remarks on the behaviour of financial market efficiency during the COVID-19 pandemic. The case of VIX," Finance Research Letters, vol. 43, Article ID 101967, 2021.

[11] P. Ferreira, "Dynamic long-range dependences in the Swiss stock market," Empirical Economics, vol. 58, no. 4, pp. 1541-1573, 2020.

[12] E. Bouri, L. A. Gil-Alana, R. Gupta, and D. Roubaud, "Modelling long memory volatility in the Bitcoin market: evidence of persistence and structural breaks," International Journal of Finance \& Economics, vol. 24, no. 1, pp. 412-426, 2019.

[13] V. Plakandaras, R. Gupta, and M. E. Wohar, "Persistence of economic uncertainty: a comprehensive analysis," Applied Economics, vol. 51, no. 41, pp. 4477-4498, 2019.

[14] G. C. Aye, M. Balcilar, R. Gupta, N. Kilimani, A. Nakumuryango, and S. Redford, "Predicting BRICS stock returns using ARFIMA models," Applied Financial Economics, vol. 24, no. 17, pp. 1159-1166, 2014.

[15] G. Bekaert and M. Hoerova, "The VIX, the variance premium and stock market volatility," Journal of Econometrics, vol. 183, no. 2, pp. 181-192, 2014.

[16] K. V. Chow, W. Jiang, B. Li, and J. Li, "Decomposing the VIX: implications for the predictability of stock returns," Financial Review, vol. 55, no. 4, pp. 645-668, 2020. 
[17] J. R. M. Hosking, "Fractional differencing," Biometrika, vol. 68 , no. 1, pp. 165-176, 1981.

[18] P. M. Robinson, "Gaussian semi-parametric estimation of long-range dependence," Annals of Statistics, vol. 23, pp. 1630-1661, 1995.

[19] K. Torre, D. Delignières, and L. Lemoine, "Detection of longrange dependence and estimation of fractal exponents through ARFIMA modelling," British Journal of Mathematical and Statistical Psychology, vol. 60, no. 1, pp. 85-106, 2007.

[20] T. Gneiting, H. Ševčíková, and D. B. Percival, "Estimators of fractal dimension: assessing the roughness of time series and spatial data," Statistical Science, vol. 27, no. 2, pp. 247-277, 2012a, https://doi.org/10.1214/11-STS370.

[21] J. M. Belbute and A. M. Pereira, "Do global CO2 emissions from fossil-fuel consumption exhibit long memory? a fractional-integration analysis," Applied Economics, vol. 49, no. 40, pp. 4055-4070, 2017, https://doi.org/10.1080/ 00036846.2016 .1273508 .

[22] A. M. Pereira and J. M. Belbute, "Final energy demand in Portugal: how persistent it is and why it matters for environmental policy," International Economic Journal, vol. 28, no. 4, pp. 661-677, 2014, https://doi.org/10.1080/10168737. 2014.920896. 